\title{
MS36-03 | Characterisation of “Polyamorphism” and the Molecular Origins of Disorder using Complementary Methods
}

Skotnicki, Marcin (School of Pharmacy and Pharmaceutical Sciences, Dublin); Hodgkinson, Paul (Durham University, Durham, GBR)

Valsartan is a widely used antihypertensive drug marketed in an "amorphous" form. DSC studies show, however, that this form is distinct from a truly amorphous material produced by quench cooling from the melt. Powder diffraction and total scattering measurements show differences implying that the "as received" material has a greater degree of ordering, but neither material gives Bragg diffraction peaks. Counter-intuitively the more ordered material has a significantly higher solubility. Solid-state NMR is a powerful adjunct to diffraction-based techniques for the characterisation of pharmaceutical materials, particularly for characterising disorder. We used a combination of time-modulated DSC, PXRD and solid-state NMR to understand the molecular origin of this "polyamorphic" behaviour in terms of conformational "defects" [1]. Other examples are presented using cocrystals and solvates of pharmaceutical actives where quantum chemical calculations are invaluable in determining whether a disordered or an ordered structure is adopted [2,3]. Understanding why such disordered materials may be intrinsically stable is important in overcoming wariness about use of disordered materials as pharmaceutical forms.

[1] M. Skotnicki, D. C. Apperley, J. A. Aguilar, B. Milanowski, M. Pyda and P. Hodgkinson, Mol. Pharmaceutics 13 (2016) 13

[2] H. E. Kerr, H. E. Mason, H. A. Sparkes and P. Hodgkinson, CrystEngComm 18 (2016) 6700

[3] A. Bērzinš̌ and P. Hodgkinson, Solid State Nucl. Magn, Reson. 65 (2015) 12 